\title{
Chigger mites (Acari: Trombiculidae) from wild birds in Costa Rica, with a description of three new species
}

\author{
Alexandr A. Stekol'nikov ${ }^{1}$, Ivan Literák ${ }^{2}$, Miroslav Čapek ${ }^{3}$ and Martin Havlíček ${ }^{4}$ \\ ${ }^{1}$ Zoological Institute, Russian Academy of Sciences, Universitetskaya 1, Saint-Petersburg 199034, Russia; \\ ${ }^{2}$ Department of Biology and Wildlife Diseases, Faculty of Veterinary Hygiene and Ecology, University of Veterinary and \\ Pharmaceutical Sciences, Palackého 1-3, 61242 Brno, Czech Republic; \\ ${ }^{3}$ Department of Avian Ecology, Institute of Vertebrate Biology, Academy of Sciences of the Czech Republic, Květná 8 , \\ 60365 Brno, Czech Republic; \\ ${ }^{4}$ Veterinary Teaching Hospital, School of Veterinary Science and Animal Production, University of Queensland, St Lucia, \\ Queensland 4072, Australia
}

Key words: Trombiculidae, Eutrombicula, new species, Costa Rica, avian parasites, taxonomy

\begin{abstract}
Three new species of chigger mites, Eutrombicula costaricensis sp. n., Eutrombicula passerinorum sp. n., and Eutrombicula hectochaeta sp. n. are described from wild birds from Costa Rica. Two species, Eutrombicula pacae (Floch et Fauran, 1957) and Parasecia fundata (Brennan, 1969), are recorded for the first time in Costa Rica and on new host species. Data on the distribution of Blankaartia sinnamaryi (Floch et Fauran, 1956) in Costa Rica are also reported.
\end{abstract}

Chigger mites were extensively collected in Costa Rica during expeditions of the Los Angeles County Museum of Natural History in 1962-1964. Results of these studies were published in two papers: Geest and Loomis (1968) recorded 13 chigger species of the genus Pseudoschoengastia Lipovsky, 1951, including 9 new species, from 15 host species of rodents and one opossum, and Webb and Loomis (1971) recorded 5 species of the genus Microtrombicula Ewing, 1950, including 3 new species, parasitizing bats and rodents. At that time, Arnold (1970) published a brief report on the finding of 7 chigger species on Costa Rican birds. Later, another species of chiggers, Hoffmanniella transylvanica Goff, 1988, was described from a vampire bat collected in Costa Rica (Goff 1988).

We report here data on six species of chigger mites found on birds in Costa Rica in 2004, including descriptions of three new species of the genus Eutrombicula Ewing, 1938.

\section{MATERIALS AND METHODS}

The fieldwork was performed in two localities on the Carribean slope of the Cordillera de Talamanca mountain range (south-eastern Costa Rica): Hitoy Cerere Biological Reserve $\left(9^{\circ} 40^{\prime} \mathrm{N}, 85^{\circ} 05^{\prime} \mathrm{W}\right)$ and Barbilla National Park $\left(9^{\circ} 59^{\prime} \mathrm{N}\right.$, $\left.85^{\circ} 27^{\prime} \mathrm{W}\right)$. These two localities are approximately $60 \mathrm{~km}$ apart and differ in elevation and habitat types. In Hitoy Cerere BR, birds were captured in a lowland rainforest on two sites: a secondary forest at an elevation of approximately $100 \mathrm{~m}$ and a primary forest at an elevation of approximately $120 \mathrm{~m}$. The study site near Barbilla NP (approximately $800 \mathrm{~m}$ from the park boundary) was a narrow strip of a water-logged pasture at an elevation of approximately $570 \mathrm{~m}$, with only scattered trees due to intensive grazing, burning and cultivation. The pasture was flanked by a secondary growth with remnants of destroyed forest; most of the adjacent national park is covered by lowland tropical rainforest. For detailed description of the localities see Sychra et al. (2006).

Birds were captured during the rainy season of 2004, in the following periods: 17-24 August (Hitoy Cerere BR, secondary forest), 24-31 August (Hitoy Cerere BR, primary forest) and 2-11 September (pasture near Barbilla NP). At these sites, a dawn-to-dusk mist-netting was conducted with the aim of capturing as many bird species and individuals as possible, and to examine them for the presence of ectoparasites. A line of about $100 \mathrm{~m}$ of mist nets was checked at least once an hour. Every individual bird was identified, sexed and aged as described elsewhere (Sychra et al. 2006). Identification of birds has been made by M. Čapek. The birds were released immediately after the examination and parasite collection.

Chiggers were preserved in $96 \%$ alcohol and later mounted in Faure-Berlese's medium. All measurements are given in micrometres $(\mu \mathrm{m})$. Terminology follows Goff et al. (1982), with some adaptation: ventral setae $(\mathrm{V})$ - setae on the ventral surface of idiosoma excluding coxal and sternal setae; VS number of ventral setae; D - dorsal idiosomal setae; DS number of dorsal idiosomal and humeral setae; TaIII - length of leg III tarsus; TaW - width of leg III tarsus; $\mathrm{m}$ - $\mathrm{t}$ - ratio between distance from mastitarsala to the base of leg III tarsus and length of leg III tarsus. Type specimens are deposited in the acarological collections of the Zoological Institute of the Russian Academy of Sciences, Saint-Petersburg (ZIN) and the Institute of Parasitology, Academy of Sciences of the Czech Republic, České Budějovice (PaÚ). 


\section{RESULTS AND DISCUSSION}

A total of 530 individuals of 79 bird species were examined. Their taxonomic positions are as follows: Tinamiformes: Tinamidae (1 species); Columbiformes: Columbidae (2); Strigiformes: Caprimulgidae (1); Trochiliformes: Trochilidae (9); Trogoniformes: Trogonidae (1); Coraciiformes: Alcedinidae (2); Galbuliformes: Bucconidae (1); Piciformes: Picidae (1); Ciconiiformes: Cathartidae (1), Accipitridae (1); Passeriformes: Furnariidae (3), Dendrocolaptidae (5), Thamnophilidae (8), Formicariidae (1), Pipridae (2), Tyrannidae (10), Troglodytidae (5), Turdidae (2), Polioptilidae (2), Vireonidae (1), Parulidae (2), Thraupidae (8), Emberizidae (4), Cardinalidae (4), Icteridae (2).

Of the 79 bird species examined, 25 were infested with six chigger species in both locations. The chiggers were found mainly on passerines. Only 2 of 20 nonpasserine species, Trogon rufus Gmelin, 1788 and Melanerpes pucherani (Malherbe, 1849), proved to be infested, whereas 23 of 59 passerine species were infested. Meanwhile, the average number of bird specimens examined of each nonpasserine species was not lesser than that of passerine birds (mean 8, median 3, and mean rank 41.9 versus $6.3,3$, and 39.4, respectively). Thus, this predominance of passerines among bird hosts of the chiggers is doubtless.

The fact that Glyphorhynchus spirurus (Vieillot, 1819) was infested with four chigger species underlines its special position as a host.

In Hitoy Cerere BR, chiggers were found on 39 of 354 (prevalence 11\%) birds examined. In Barbilla NP, they were found on 34 of 176 (prevalence 19\%) birds. The conditions of the environment in the pasture near Barbilla NP make the presence of trombiculids improbable in that place, first of all, since the water-logged ground can hardly be considered as a suitable biotope for soil-inhabiting nymphs and adults of these mites. At the same time, the birds captured in the pasture inhabited neighbouring secondary forest where they could have been attacked by chiggers. Thus, probably all the chiggers collected in both localities are inhabitants of forest. Possible biotopes of post-larval free-living instars for these species can be suggested by considering chigger species distribution among hosts and the biology of these hosts, as follows.

\section{Eutrombicula costaricensis sp. n.}

Figs. 1-11

Diagnosis. $\mathrm{SIF}=7 \mathrm{BS}-\mathrm{N}-2-3111.1000 ; \mathrm{fPp}=$ $\mathrm{B} / \mathrm{B} / \mathrm{NNB} ; \mathrm{fCx}=1.1 .1 ; \mathrm{fSt}=2.2 ; \mathrm{fSc}: \mathrm{PL}>\mathrm{AM}>\mathrm{AL}$; $\mathrm{Ip}=851 ; \mathrm{fD}=2 \mathrm{H}-6-6-2-4 ; \mathrm{fV}=6-2-2-2 ; \mathrm{DS}=20 ; \mathrm{VS}$ $=12 ; \mathrm{NDV}=32$.

Description. LARVA. Idiosoma. Eyes 2+2. Pair of humeral setae; 18 dorsal idiosomal setae, arranged 6-62-4 (rare variations: $6-5-2-4$ and 7-6-2-4); 2 pairs of sternal setae and 12 ventral setae, arranged 6-2-2-2 (rare variations: 5-2-2-2 and 6-2-2-3); total idiosomal setae
32. Gnathosoma. Cheliceral blade with tricuspid cap; cheliceral base with sparse large puncta; gnathobase with dense small puncta and distinct transverse striations, bearing pair of branched setae; palpal femur and genu sparsely punctate; galeala nude; palpal claw with long stout axial prong and shorter accessory prong arising medially; seta on palpal femur branched; seta on palpal genu with 1-2 branches, sometimes seems to be nude; dorsal and lateral palpal tibial setae nude, ventral palpal tibial seta branched; palpal tarsus with 7 branched setae, nude medial subterminala and basal tarsala. Scutum. Nearly rectangular, with posterior margin straight in middle part, moderately punctate, without transverse striations; AM base on level of ALs; SB anterior to level of PLs; $\mathrm{PL}>\mathrm{AM}>\mathrm{AL}$; sensilla flagelliform with 7-8 branches in distal half. Legs. All 7-segmented, with pair of claws and clawlike empodium, coxae with indistinct longitudinal striations. Leg I: coxa with 1 nonspecialised branched seta (1B); trochanter 1B; basifemur $1 \mathrm{~B}$; telofemur $5 \mathrm{~B}$; genu 4B, 3 genualae, microgenuala; tibia 8B, 2 tibialae, microtibiala; tarsus 22B, tarsala 14 long, microtarsala, subterminala, parasubterminala, pretarsala. Leg II: coxa 1B; trochanter 1B; basifemur $2 \mathrm{~B}$; telofemur $4 \mathrm{~B}$; genu $3 \mathrm{~B}$, genuala; tibia $6 \mathrm{~B}, 2$ tibialae; tarsus $16 \mathrm{~B}$, tarsala 13 long, microtarsala, pretarsala. Leg III: coxa 1B; trochanter 1B; basifemur 2B; telofemur 3B; genu $3 \mathrm{~B}$, genuala; tibia $6 \mathrm{~B}$, tibiala; tarsus 14B, mastitarsala nude.

Standard measurements of the type series $(n=12)$ :

\begin{tabular}{|c|c|c|c|c|c|c|c|}
\hline & AW & PW & SB & ASB & PSB & SD \\
\hline \multicolumn{2}{|c|}{ Holotype } & 77 & 87 & 43 & 27 & 23 & 50 \\
\hline \multicolumn{2}{|c|}{ Minimum } & 74 & 84 & 40 & 23 & 23 & 48 \\
\hline \multicolumn{2}{|c|}{ Maximum } & 79 & 90 & 45 & 28 & 26 & 54 \\
\hline \multicolumn{2}{|c|}{ Mean } & 77 & 87 & 42 & 26 & 24 & 51 \\
\hline P-PL & $\mathrm{AP}$ & $\mathrm{AM}$ & $\mathrm{AL}$ & PL & $\mathrm{S}$ & $\mathrm{H}$ & $\mathrm{D}$ \\
\hline 18 & 25 & 41 & 37 & 56 & - & 56 & $45-55$ \\
\hline 17 & 23 & 41 & 35 & 49 & 59 & 47 & $41-50$ \\
\hline 20 & 27 & 44 & 41 & 61 & 61 & 56 & $50-57$ \\
\hline 18 & 24 & 42 & 37 & 55 & 60 & 54 & $45-54$ \\
\hline
\end{tabular}

\begin{tabular}{cccccccc}
\hline $\mathrm{V}$ & pa & pm & pp & Ip & TaIII & TaW & m-t \\
\hline- & 299 & 266 & 286 & 851 & 72 & 20 & 0.268 \\
$40-52$ & 290 & 256 & 283 & 833 & 68 & 18 & 0.268 \\
$40-52$ & 310 & 270 & 297 & 869 & 74 & 20 & 0.293 \\
$40-52$ & 300 & 261 & 289 & 851 & 71 & 19 & 0.278 \\
\hline
\end{tabular}

Hosts and possible biotopes: Glyphorhynchus spirurus, Xiphorhynchus susurrans (Jardine, 1847), Deconychura longicauda (Pelzen, 1868) (Passeriformes, Dendrocolaptidae). All these hosts are arboreal species foraging almost entirely on trees. Only $X$. susurrans regularly descends to the ground to take prey (del Hoyo et al. 2003). Thus, Eutrombicula costaricensis may be an arboreal chigger, inhabiting roots of epiphytes, like the Oriental and Australian species Ascoschoengastia indica (Hirst, 1915) (Wharton and Carver 1946), or tree hollows, like the European Ascoschoengastia latyshevi (Schluger, 1955) (Sixl 1969). 


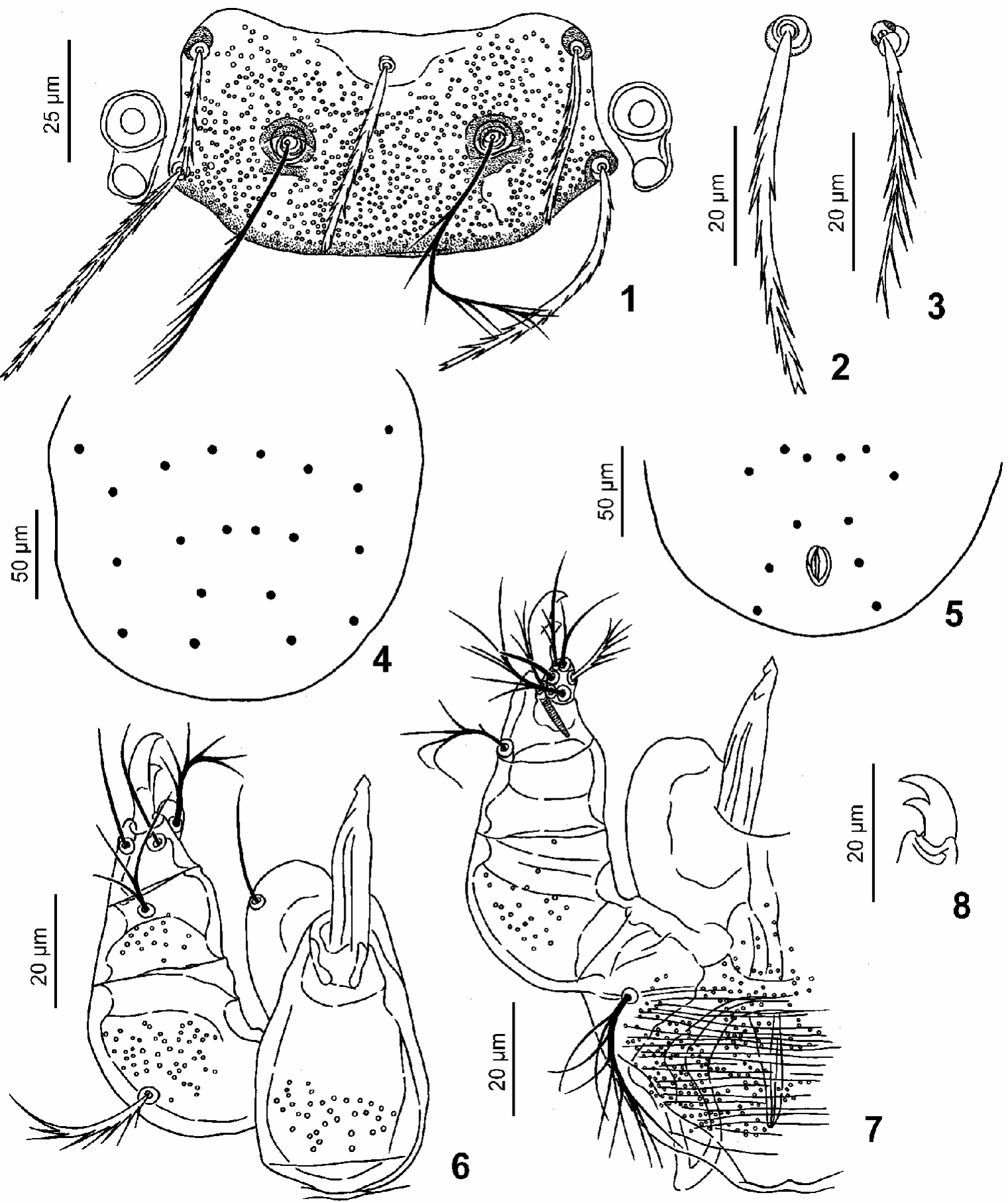

Figs. 1-8. Eutrombicula costaricensis sp. n., larva. Fig. 1. Scutum and eyes. Fig. 2. Dorsal idiosomal seta. Fig. 3. Ventral (preanal) idiosomal seta. Fig. 4. Arrangement of dorsal idiosomal setae. Fig. 5. Arrangement of ventral idiosomal setae. Fig. 6. Dorsal aspect of gnathosoma. Fig. 7. Ventral aspect of gnathosoma. Fig. 8. Palpal claw, lateral view.

T y p e d a t a : Holotype larva (no. 7113, T-Tr.-30), Barbilla NP, from D. longicauda, 3 Sept. 2004, near the vent. Two hundred and thirty-two paratype larvae: Barbilla NP, from D. longicauda, G. spirurus and X. susurrans. Additional material: 17 larvae, Barbilla NP, from G. spirurus. The holotype and 200 paratypes (nos. 7041 to 7343 ) are depos- ited in ZIN; 32 paratypes (nos. 7196 to 7344 ) are deposited in PaÚ (coll. no. PaÚ 2012).

P r e v a le n c e : D. longicauda, Barbilla NP (B): 1 positive / 1 examined; G. spirurus, Hitoy Cerere BR (HC): 0/10, B: 8/9; X. susurrans, HC: 0/4, B: $2 / 2$.

E t y $\mathrm{m}$ o $l$ o g y : Specific epithet refers to the terra typica. 


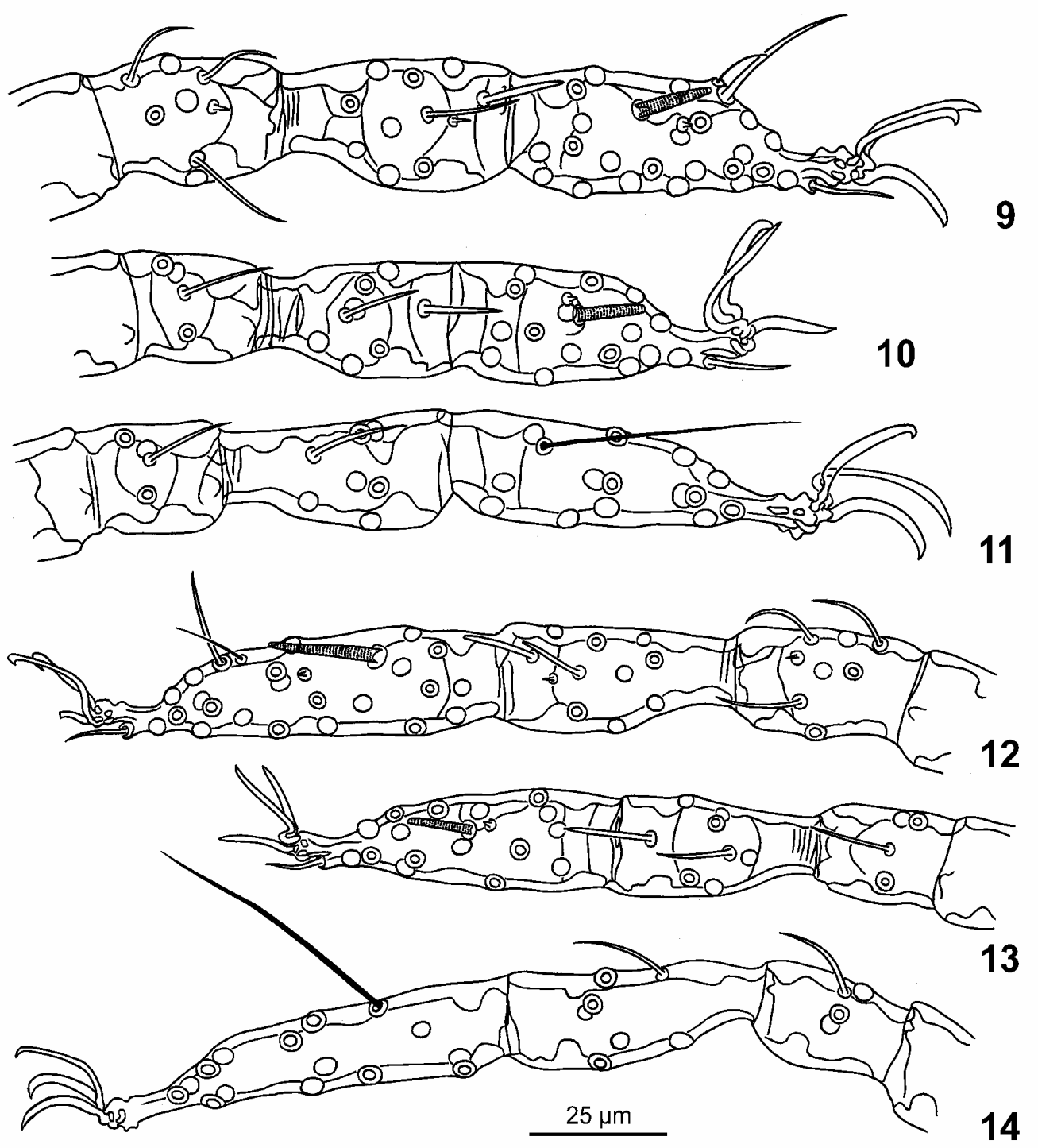

Figs. 9-11. Eutrombicula costaricensis sp. n., larva. Fig. 9. Leg I. Fig. 10. Leg II. Fig. 11. Leg III. Figs. 12-14. Eutrombicula passerinorum sp. n., larva. Fig. 12. Leg I. Fig. 13. Leg II. Fig. 14. Leg III.

Differential diagnosis. The new species is similar to Eutrombicula tachirae Brennan et Reed, 1974 but differs from it in having branched ventral palpal tibial seta $(\mathrm{fPp}=\mathrm{B} / \mathrm{B} / \mathrm{NNB}$ versus $\mathrm{B} / \mathrm{B} / \mathrm{NNN})$, much shorter scutal and idiosomal setae $(\mathrm{AM}=41-44$ versus $68, \mathrm{AL}=35-$ 41 versus $63, \mathrm{PL}=49-61$ versus $90, \mathrm{H}=47-56$ versus $78, \mathrm{D}=41-57$ versus $62-92)$, shorter legs $(\mathrm{Ip}=833-$ 869 versus 965), shorter tarsalae on legs I and II (tarsala I 14 versus 19 long, tarsala II 13 versus 17 long), and somewhat smaller scutum (AW $=74-79$ versus 84 , PW $=84-90$ versus $100, \mathrm{AP}=23-27$ versus 31$)$. The new species also resembles Eutrombicula tinami (Oudemans, 1910) but differs from it in having $\mathrm{fD}=2 \mathrm{H}-6-6-2-4$ versus $2 \mathrm{H}-6-6-2-4-2$, shorter scutum (PSB $=23-26$ versus $35, \mathrm{SD}=48-54$ versus $64, \mathrm{AP}=23-27$ versus 38$)$, and shorter scutal setae $(\mathrm{AM}=41-44$ versus $56-59, \mathrm{AL}$ $=35-41$ versus $48-53, \mathrm{PL}=49-61$ versus $62-63)$. The measurements of $E$. tinami are given by Fuller (1952).
Eutrombicula passerinorum sp. n.

Figs. $12-19$

Diagnosis. SIF $=7 \mathrm{BS}-\mathrm{N}-2-3111.1000 ; \mathrm{fPp}=\mathrm{B} / \mathrm{B} /$ $\mathrm{NNB} ; \mathrm{fCx}=1.1 .1 ; \mathrm{fSt}=2.2 ; \mathrm{fSc}: \mathrm{PL}>\mathrm{AM} \geq \mathrm{AL} ; \mathrm{Ip}=$ $874 ; \mathrm{fD}=2 \mathrm{H}-6-6-2-4 ; \mathrm{fV}=6-2-2-2 ; \mathrm{DS}=20 ; \mathrm{VS}=12$; $\mathrm{NDV}=32$.

Description. LaRVA. Idiosoma. Eyes 2+2. Pair of humeral setae; 18 dorsal idiosomal setae, arranged 6-6$2-4 ; 2$ pairs of sternal setae and 12 ventral setae, arranged 6-2-2-2; total idiosomal setae 32. Gnathosoma. Cheliceral blade with tricuspid cap; cheliceral base with dense puncta in basal part and few large puncta in middle portion; gnathobase with dense puncta and distinct transverse striations, bearing pair of branched setae; palpal femur, genu and tibia sparsely punctate; galeala nude; palpal claw with long stout axial prong and shorter accessory prong arising submedially; seta on palpal femur branched; seta on palpal genu with 1-2 


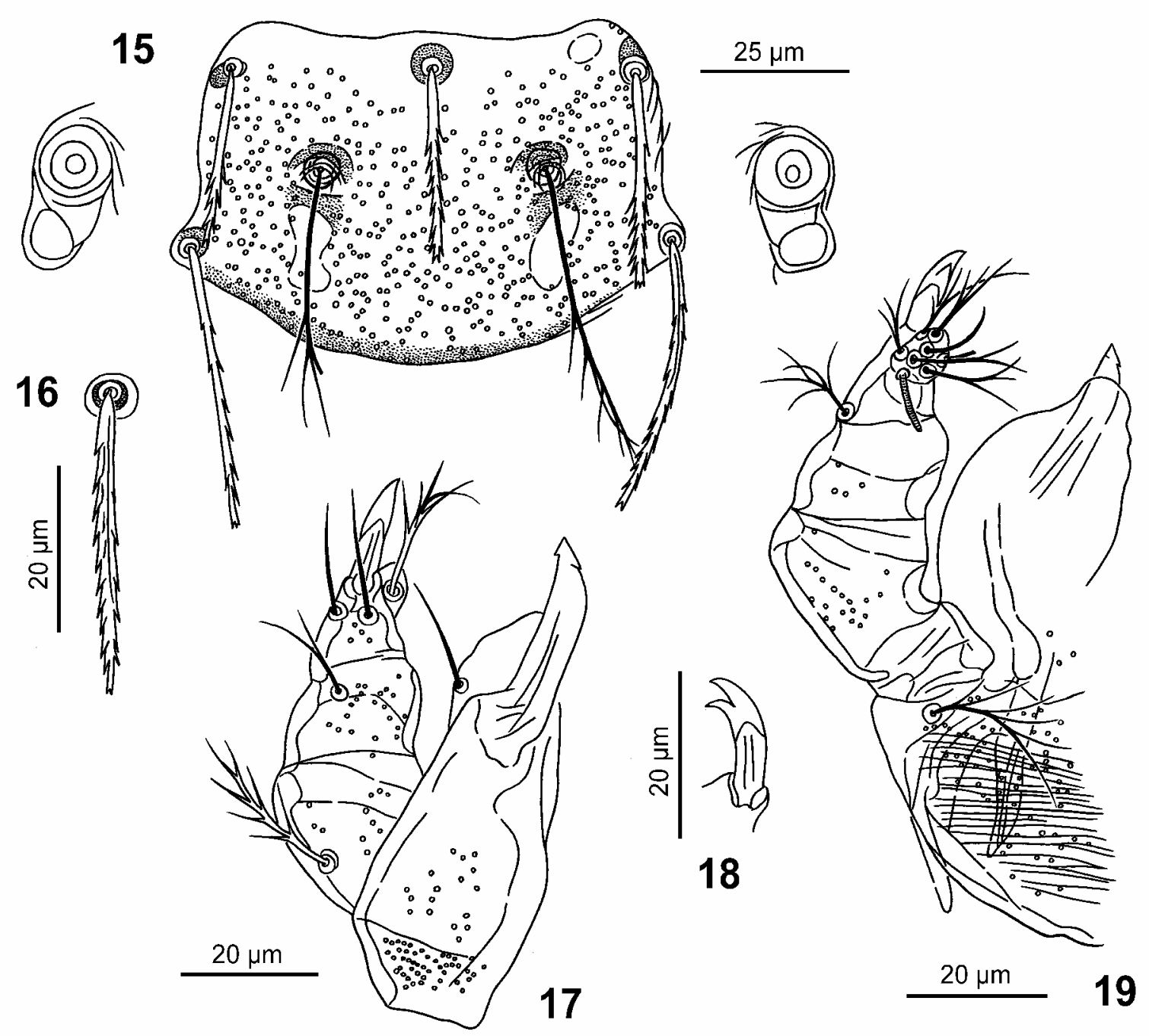

Figs. 15-19. Eutrombicula passerinorum sp. n., larva. Fig. 15. Scutum and eyes. Fig. 16. Dorsal idiosomal seta. Fig. 17. Dorsal aspect of gnathosoma. Fig. 18. Palpal claw, lateral view. Fig. 19. Ventral aspect of gnathosoma.

branches, sometimes seems to be nude; dorsal and lateral palpal tibial setae nude, ventral palpal tibial seta branched; palpal tarsus with 7 branched setae, nude curved medial subterminala and basal tarsala. Scutum. Nearly rectangular, with rounded posterior margin and rather sparse puncta, without transverse striations; AM base on level of ALs; SB far anterior to level of PLs; PL $>A M \geq A L ;$ sensilla flagelliform with about 6 branches in distal half. Legs. All 7-segmented, with pair of claws and clawlike empodium, coxae with indistinct longitudinal striations. Leg I: coxa 1B; trochanter 1B; basifemur $1 \mathrm{~B}$; telofemur $5 \mathrm{~B}$; genu $4 \mathrm{~B}, 3$ genualae, microgenuala; tibia 8B, 2 tibialae, microtibiala; tarsus $22 \mathrm{~B}$, tarsala 20-21 long, microtarsala, subterminala, parasubterminala, pretarsala. Leg II: coxa 1B; trochanter 1B; basifemur $2 \mathrm{~B}$; telofemur $4 \mathrm{~B}$; genu $3 \mathrm{~B}$, genuala; tibia 6B, 2 tibialae; tarsus 16B, tarsala 12 long, microtarsala, pretarsala. Leg III: coxa 1B; trochanter $1 \mathrm{~B}$; basifemur $2 \mathrm{~B}$; telofemur $3 \mathrm{~B}$; genu $3 \mathrm{~B}$, genuala; tibia $6 \mathrm{~B}$, tibiala; tarsus 14B, mastitarsala nude.
Standard measurements of the type series $(n=3)$ :

\begin{tabular}{l|ccccccc}
\hline & AW & PW & SB & ASB & PSB & SD & P-PL \\
\hline Holotype & 68 & 82 & 39 & 25 & 33 & 58 & 21 \\
Paratype & 68 & 79 & 37 & 25 & 31 & 56 & 20 \\
Paratype & 65 & 78 & 37 & 24 & 30 & 55 & 20 \\
\hline
\end{tabular}

\begin{tabular}{cccccccc}
\hline AP & AM & AL & PL & S & H & D & V \\
\hline $29 / 31$ & 33 & $31 / 37$ & $47 / 48$ & 51 & $45 / 47$ & $36-43$ & $35-43$ \\
29 & 29 & $26 / 28$ & $42 / 43$ & - & 41 & $33-40$ & $31-40$ \\
27 & 31 & 25 & $41 / 44$ & - & $40 / 42$ & $34-43$ & - \\
\hline \multicolumn{1}{c}{ pa } & pm & pp & Ip & TaIII & TaW & m-t \\
\hline- & - & - & - & 81 & 15 & 0.316 \\
315 & 263 & 310 & 887 & 78 & 15 & 0.315 \\
310 & 256 & 295 & 860 & 74 & 14 & 0.324 \\
\hline
\end{tabular}

Hosts and possible biotopes:Ramphocaenus melanurus Vieillot, 1819 (Passeriformes, Polioptilidae), and Ramphocelus passerinii Bonaparte, 1831 (Passeriformes, Thraupidae). Both these species forage by inspecting foliage, tree branches and leaf litter accumulated in epiphytes. Only $R$. passerinii sometimes descend to the ground (Stiles and Scutch 1989). Thus, soil microbiotopes 
located on trees are at least one of possible habitats of the Eutrombicula passerinorum postlarval instars.

T y p e d a t a : Holotype larva (no. 7037, T-Tr.-31), Hitoy Cerere BR, from Ramphocaenus melanurus, 23 Aug. 2004. Two paratype larvae: Hitoy Cerere BR, from the uropygeum of Ramphocelus passerinii. The holotype and one paratype (no. 7080) are deposited in ZIN; one paratype (no. 7081) is deposited in PaÚ (coll. no. PaÚ 2013).

P r e v a 1 e n c e : Ramphocaenus melanurus, HC: $1 / 5$; Ramphocelus passerinii, HC: 1/3, B: 0/7.

E t y m o lo g y : Specific epithet derives from order name of the hosts.

Differential diagnosis. The new species is similar to Eutrombicula tinami (Oudemans, 1910) but differs from it in having $\mathrm{fD}=2 \mathrm{H}-6-6-2-4$ versus $2 \mathrm{H}-6-6-2-4-2$, much shorter scutal and idiosomal setae $(\mathrm{AM}=29-33$ versus $56-59, \mathrm{AL}=25-37$ versus $48-53, \mathrm{PL}=41-48$ versus $62-63, \mathrm{D}=33-43$ versus $53-56)$, and smaller scutum $(\mathrm{AW}=65-68$ versus $76-77, \mathrm{PW}=78-82$ versus 92, $\mathrm{ASB}=24-25$ versus $29, \mathrm{PSB}=30-33$ versus $35, \mathrm{SD}=$ 55-58 versus 64, AP $=27-31$ versus 38 ).

\section{Eutrombicula hectochaeta sp. n.}

Figs. 20-30

Diagnosis. SIF $=7 \mathrm{BS}-\mathrm{N}-2-3111.1000 ; \mathrm{fPp}=\mathrm{B} / \mathrm{N} /$ $\mathrm{NNN}$; $\mathrm{fCx}=1.1 .1$; $\mathrm{fSt}=2.2$; fSc: $\mathrm{PL}>\mathrm{AM}>\mathrm{AL}$; $\mathrm{Ip}=$ 933; fD = 2H-6-(11-13)-(10-13)-(10-14)-..; fV = (6$7)-(28-39) ; \mathrm{DS}=52-64 ; \mathrm{VS}=34-46 ; \mathrm{NDV}=86-103$.

Description. LARVA. Idiosoma. Eyes 2+2. Pair of humeral setae; 50-62 dorsal idiosomal setae; in 1st posthumeral row 6 setae, rarely 7, in 2nd row 9-16 setae, usually $11-13$, in 3rd row 8-13 setae, usually 10 13 , in 4 th row 10-14 setae; 2 pairs of sternal setae and 34-46 ventral setae, including isolated group of anterior 6-7 setae; total idiosomal setae 86-103. Gnathosoma. Cheliceral blade with tricuspid cap; cheliceral base with dense small puncta in basal part and sparse large puncta in middle portion; gnathobase with dense puncta and distinct transverse striations, bearing pair of branched setae; palpal femur and genu punctate; galeala nude; palpal claw with long stout axial prong and shorter accessory prong arising medially; seta on palpal femur branched; seta on palpal genu nude; dorsal, lateral, and ventral palpal tibial setae nude; palpal tarsus with 7 branched setae, nude curved medial subterminala and basal tarsala. Scutum. Nearly rectangular, with posterior margin straight in middle part, dense small puncta, without transverse striations; AM base on level of ALs; $\mathrm{SB}$ far anterior to level of PLs; $\mathrm{PL}>\mathrm{AM}>\mathrm{AL}$; sensilla flagelliform with about 8 branches in distal half. Legs. All 7-segmented, with pair of claws and clawlike empodium, coxae with indistinct longitudinal striations. Leg $\mathrm{I}$ : coxa $1 \mathrm{~B}$; trochanter $1 \mathrm{~B}$; basifemur $1 \mathrm{~B}$; telofemur $5 \mathrm{~B}$; genu 4B, 3 genualae, microgenuala; tibia $8 \mathrm{~B}, 2$ tibialae, microtibiala; tarsus 22B, tarsala 14 long, microtarsala, subterminala, parasubterminala, pretarsala. Leg II: coxa $1 \mathrm{~B}$; trochanter $1 \mathrm{~B}$; basifemur $2 \mathrm{~B}$; telofemur $4 \mathrm{~B}$; genu
3B, genuala; tibia 6B, 2 tibialae; tarsus 16B, tarsala 10 11 long, microtarsala, pretarsala. Leg III: coxa $1 \mathrm{~B}$; trochanter $1 \mathrm{~B}$; basifemur $2 \mathrm{~B}$; telofemur $3 \mathrm{~B}$; genu $3 \mathrm{~B}$, genuala; tibia $6 \mathrm{~B}$, tibiala; tarsus $14 \mathrm{~B}$, mastitarsala nude.

Standard measurements of the type series $(n=9)$ :

\begin{tabular}{|c|c|c|c|c|c|c|}
\hline & AW & PW & SB & ASB & PSB \\
\hline \multicolumn{2}{|c|}{ Holotype } & 77 & 89 & 42 & 29 & 27 \\
\hline \multicolumn{2}{|c|}{ Minimum } & 73 & 86 & 40 & 28 & 27 \\
\hline \multicolumn{2}{|c|}{ Maximum } & 80 & 93 & 44 & 31 & 30 \\
\hline \multicolumn{2}{|c|}{ Mean } & 76 & 90 & 43 & 29 & 29 \\
\hline SD & P-PL & AP & AM & $\mathrm{AL}$ & PL & $\mathrm{S}$ \\
\hline 56 & 18 & 29 & 39 & 37 & 54 & - \\
\hline 56 & 16 & 28 & 39 & 33 & 50 & 59 \\
\hline 60 & 21 & 31 & 47 & 40 & 58 & 59 \\
\hline 57 & 18 & 29 & 43 & 36 & 54 & 59 \\
\hline $\mathrm{H}$ & $\mathrm{D}$ & $\mathrm{V}$ & $\mathrm{pa}$ & $\mathrm{pm}$ & $\mathrm{pp}$ & Ip \\
\hline 52 & $33-53$ & $32-47$ & 326 & 286 & 308 & 920 \\
\hline 51 & $32-50$ & $32-47$ & 326 & 275 & 306 & 918 \\
\hline 59 & $38-57$ & $41-50$ & 344 & 290 & 333 & 954 \\
\hline 55 & $34-54$ & $36-49$ & 336 & 284 & 313 & 933 \\
\hline DS & VS & TaI & & & TaW & $\mathrm{m}-\mathrm{t}$ \\
\hline 62 & 34 & 77 & & & 18 & 0.244 \\
\hline 52 & 34 & 76 & & & 18 & 0.242 \\
\hline 64 & 46 & 79 & & & 18 & 0.260 \\
\hline 58 & 38 & 78 & & & 18 & 0.247 \\
\hline
\end{tabular}

Hosts and possible biotopes: Glyphorhynchus spirurus (Vieillot, 1819) (Passeriformes, Dendrocolaptidae). This species inhabits trees and forages mainly by inspecting trunks and branches at varying heights above the ground (Stiles and Scutch 1989, del Hoyo et al. 2003). Thus, Eutrombicula hectochaeta probably is arboreal species too.

T y p e d a t a: Holotype larva (no. 7250, T-Tr.-32), Barbilla NP, from G. spirurus, 2 Sept. 2004, around the vent. Fiftyfour paratype larvae: Barbilla NP and Hitoy Cerere BR, from $G$. spirurus. Additional material: 25 provisionally identified larvae in alcohol, deposited in ZIN, collected in Hitoy Cerere BR, 28 Aug. 2004, from G. spirurus. The holotype and 30 paratypes (nos. 7056 to 7272 ) are deposited in ZIN; 24 paratypes (nos. 7055 to 7323) are deposited in PaÚ (coll. no. PaÚ 2014).

Prevale n c e: G. spirurus, HC: 2/10, B: 6/9.

E t y m o 1 o g y : Specific epithet refers to the presence of about 100 idiosomal setae in this species.

Differential diagnosis. The new species is similar to Eutrombicula variabilis Brennan et Reed, 1974 but differs from it in having much more numerous idiosomal setae (DS $=52-64$ versus $30-35, \mathrm{VS}=34-46$ versus 25 , $\mathrm{NDV}=86-103$ versus $40-60)$, narrower scutum (AW $=$ $73-80$ versus $82, \mathrm{PW}=86-93$ versus $103, \mathrm{SB}=40-44$ versus 50), shorter scutal and idiosomal setae $(\mathrm{AM}=$ $39-47$ versus $64, \mathrm{AL}=33-40$ versus $55, \mathrm{PL}=50-58$ versus $74, \mathrm{H}=51-59$ versus $73, \mathrm{D}=32-57$ versus $55-$ $70)$, longer legs $(\mathrm{Ip}=918-954$ versus 785$)$, and shorter tarsala on leg II (10-11 versus 15 long). 


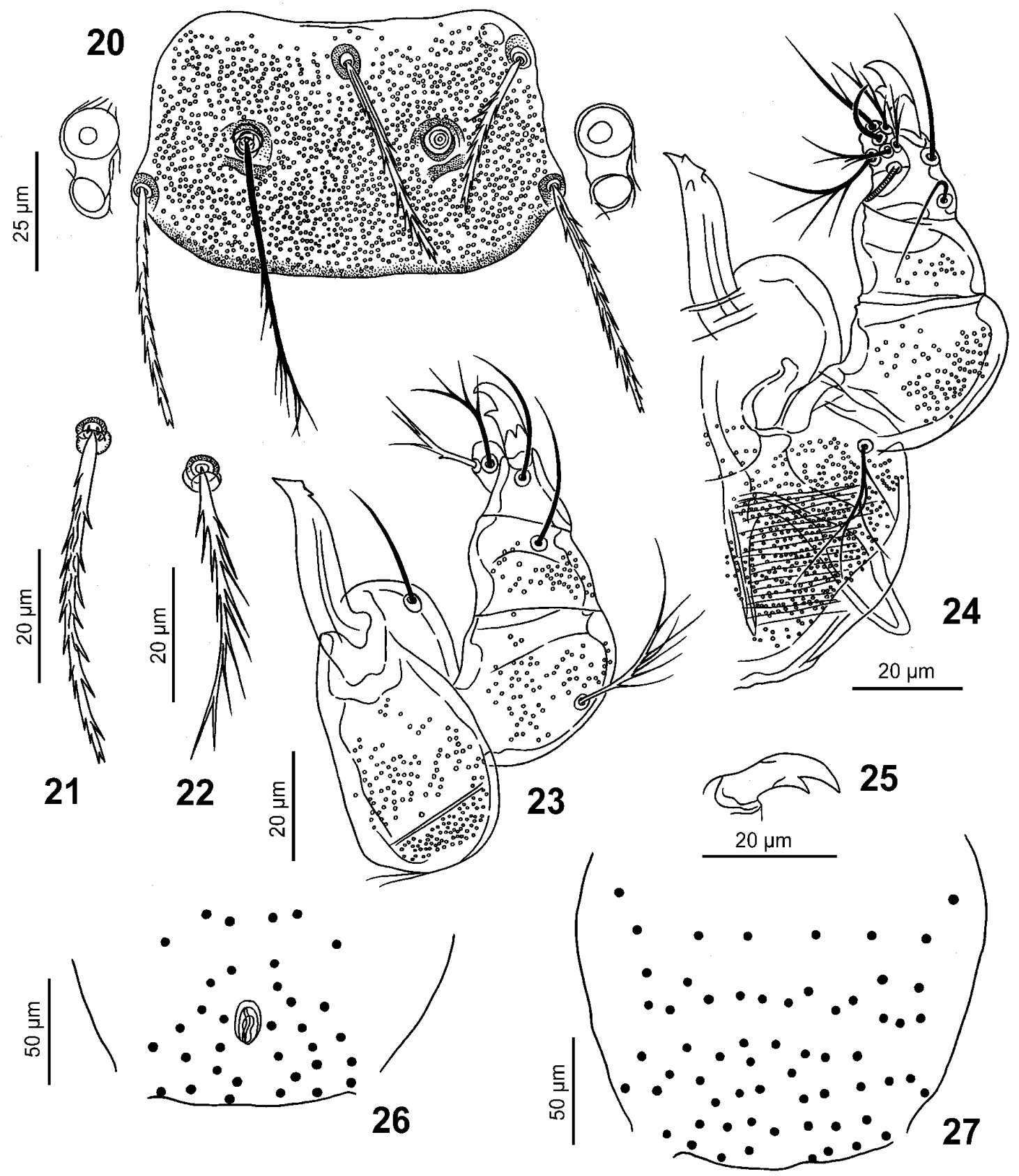

Figs. 20-27. Eutrombicula hectochaeta sp. n., larva. Fig. 20. Scutum and eyes. Fig. 21. Dorsal idiosomal seta. Fig. 22. Ventral (preanal) idiosomal seta. Fig. 23. Dorsal aspect of gnathosoma. Fig. 24. Ventral aspect of gnathosoma. Fig. 25. Palpal claw, lateral view. Fig. 26. Arrangement of ventral idiosomal setae. Fig. 27. Arrangement of dorsal idiosomal setae.

Eutrombicula pacae (Floch et Fauran, 1957)

Standard measurements $(n=3)$ :

\begin{tabular}{l|ccccccc}
\hline & AW & PW & SB & ASB & PSB & SD & P-PL \\
\hline Minimum & 63 & 74 & 34 & 23 & 26 & 48 & 18 \\
Maximum & 67 & 77 & 36 & 23 & 27 & 50 & 18 \\
Mean & 65 & 76 & 35 & 23 & 26 & 49 & 18 \\
\hline
\end{tabular}

\begin{tabular}{ccccccc}
\hline pa & pm & pp & Ip & TaIII & TaW & m-t \\
\hline 279 & 229 & 256 & 763 & 63 & 15 & 0.200 \\
281 & 230 & 261 & 772 & 65 & 15 & 0.222 \\
280 & 230 & 258 & 768 & 64 & 15 & 0.211 \\
\hline
\end{tabular}

\begin{tabular}{ccccccc}
\hline AP & AM & AL & PL & H & D & V \\
\hline 24 & 45 & 28 & 43 & 42 & $36-41$ & $31-38$ \\
26 & 45 & 34 & 49 & 45 & $38-43$ & $32-39$ \\
25 & 45 & 32 & 46 & 44 & $37-42$ & $32-38$
\end{tabular}

Hosts and possible bi otopes:Formicarius analis (d'Orbigny et Lafresnaye, 1837) (Passeriformes, Formicariidae) and Henicorhina leucosticta (Cabanis, 


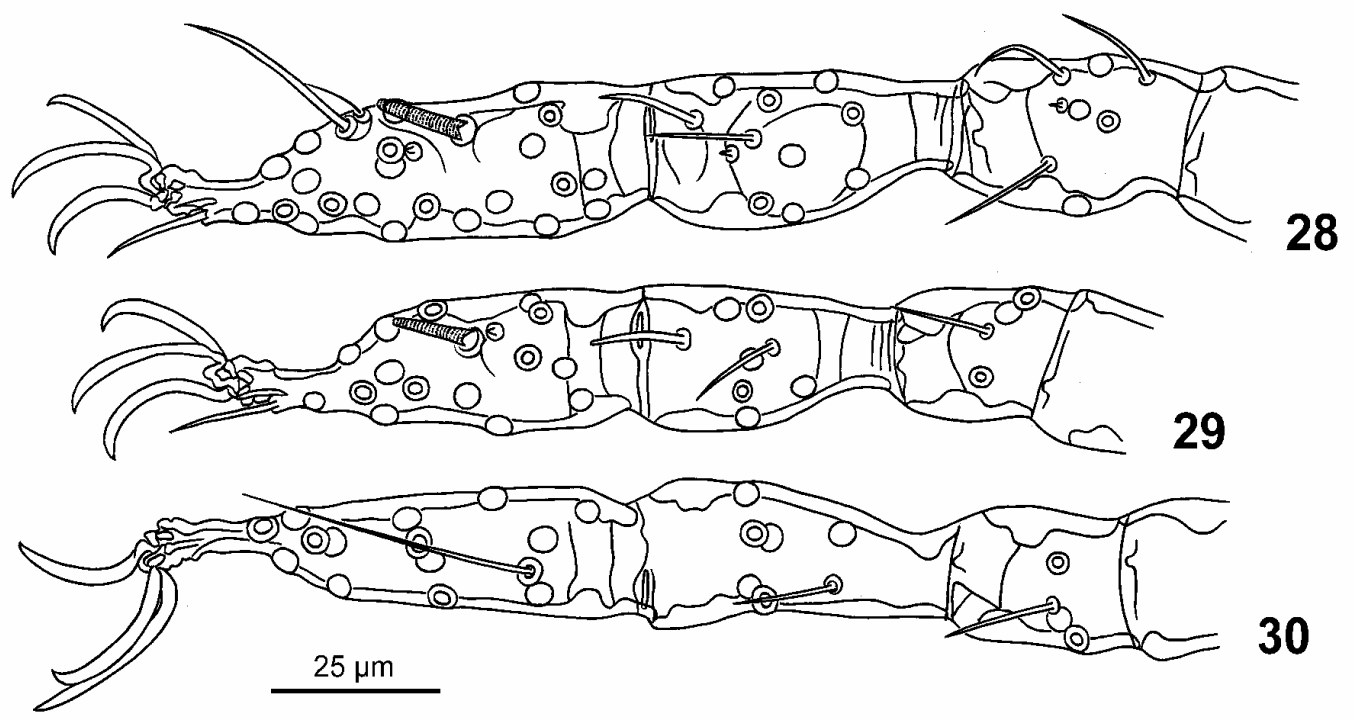

Figs. 28-30. Eutrombicula hectochaeta sp. n., larva. Fig. 28. Leg I. Fig. 29. Leg II. Fig. 30. Leg III.

1847) (Passeriformes, Troglodytidae), new host records. Previously reported hosts: Agouti paca (Linnaeus, 1766) (Rodentia, Agoutidae) (type host), Dasyprocta leporina (Linnaeus, 1758) (Rodentia, Dasyproctidae), Proechimys cayennensis (Desmarest, 1817), P. semispinosus (Tomes, 1860) (Rodentia, Echimyidae), Rhipidomys sp. (Rodentia, Muridae), Caluromys philander (Linnaeus, 1758), Marmosa fuscata Thomas, 1896, M. robinsoni Bangs, 1898 (Marsupialia, Didelphidae), Artibeus lituratus (Olfers, 1818), Carollia brevicauda (Schinz, 1821) (Chiroptera, Phyllostomidae), Turdus leucomelas Vieillot, 1818 (Passeriformes, Turdidae) (Floch and Fauran 1957, Brennan and Reed 1974, Brennan and Bronswijk 1975). This list includes both terrestrial and arboreal host species. The paca Agouti paca, Brazilian agouti Dasyprocta leporina and Proechimys spiny rats are terrestrial species. Both bird hosts of Eutrombicula pacae recorded in our study, Formicarius analis and Henicorhina leucosticta, forage mainly on or near the forest floor (Stiles and Scutch 1989, del Hoyo et al. 2003, 2005). On the other hand, bare-tailed woolly opossum Caluromys philander and phyllostomid bats are arboreal animals. These data suggest a wide biotopic distribution of Eutrombicula pacae.

D i s tri b u t i o n : French Guiana (Floch and Fauran 1957), Venezuela (Brennan and Reed 1974), Surinam (Brennan and Bronswijk 1975). Recorded in Costa Rica for the first time.

M a t e r i a 1 e x a m i n e d: 1 larva, Barbilla NP, from $H$. leucosticta; 16 larvae, Hitoy Cerere BR, from $F$. analis, on the abdomen, around the vent.

Pre vale n c e : H. leucosticta, B: $1 / 2 ; F$. analis, HC: $1 / 4$.

Remarks. As compared with the original description, our specimens have the scutum straight in the middle part of the posterior margin versus angulate, somewhat larger AW (63-67 versus 58-64), shorter AL (28-34 versus 31-40) and PL (43-49 versus 48-55).

\section{Parasecia fundata (Brennan, 1969)}

Host s and possible bi oto pes: Melanerpes pucherani (Piciformes, Picidae) and Xiphorhynchus susurrans (Passeriformes, Dendrocolaptidae), new host records. Previous host records (Brennan 1969): Caluromys philander (type host), Didelphis marsupialis Linnaeus, 1758 (Marsupialia, Didelphidae), Proechimys cayennensis, Glyphorhynchus spirurus. The occurrence of Parasecia fundata both on terrestrial mammals (Proechimys spiny rats and southern opossum Didelphis marsupialis) and on arboreal host species (birds Glyphorhynchus spirurus and Melanerpes pucherani, opossum Caluromys philander) is the evidence of its wide biotopic distribution. The biology of M. pucherani is similar to G. spirurus (Stiles and Scutch 1989, del Hoyo et al. 2002).

D i s t r i b u t i o n : NE Brazil (Brennan 1969). Recorded in Costa Rica for the first time.

M a t e r i a 1 e x a m in e d : 1 larva, Barbilla NP, from $M$. pucherani; 82 larvae, Barbilla NP and Hitoy Cerere BR, from $X$. susurrans and G. spirurus.

P r e v a 1 e n c e : M. pucherani, B: $1 / 1 ; X$. susurrans, HC: 2/4, B: 1/2; G. spirurus, HC: 1/10, B: 4/9.

\section{Blankaartia sinnamaryi (Floch et Fauran, 1956)}

Hosts and possible biotopes: Many species of birds, also phyllostomid bats (Brennan and Yunker 1966) and reptiles (Brennan and Jones 1960). A variety of hosts inhabiting different levels of forest suggests a wide biotopic distribution of $B$. sinnamaryi.

D i s t r i b u t i o n : French Guiana (Floch and Fauran 1956), Texas, Florida, Panama, Costa Rica, Surinam, Peru, Jamaica, Trinidad, Cuba. The species was previously recorded on 8 bird species in Costa Rica by Arnold (1970).

Material examined ( p revale n ce) : 284 larvae, from: $\mathrm{P}$ a s s e $\mathrm{r}$ i f o r m e s : Attila spadiceus (Gmelin, 1789) (HC: 1/1, B: 1/2), Oncostoma cinereigulare 
(Sclater, 1857) (HC: 2/3), Pitangus sulphuratus (Linnaeus, 1766) (HC: 1/1), Tyrannus melancholicus Vieillot, 1819 (HC: 1/1) (Tyrannidae), Cyanocompsa cyanoides (Lafresnaye, 1847) (HC: 2/9, B: 0/3) (Cardinalidae), Cymbilaimus lineatus (Leach, 1814) (B: 1/1), Myrmotherula fulviventris (Lawrence, 1862) (HC: 2/3, B: 1/1), Thamnistes anabatinus Sclater et Salvin, 1860 (B: 1/2) (Thamnophilidae), Microbates cinereiventris (Sclater, 1855) (HC: 3/3) (Polioptilidae), Oryzoborus funereus (Sclater, 1859) (HC: 11/30, B: 4/7), Sporophila americana (=aurita) (Gmelin, 1789) (HC: 6/23, B: 4/6), Volatinia jacarina (Linnaeus, 1766) (B: 1/3) (Emberizidae), Pipra mentalis Sclater, 1857 (HC: 1/8, B: 1/2) (Pipridae), Seiurus motacilla (Vieillot, 1808) (B: 1/4) (Parulidae), Ramphocelus passerinii (HC: 0/3, B: 1/7), Tachyphonus delattrii Lafresnaye, 1847 (HC: 0/7, B: 3/15) (Thraupidae), Henicorhina leucosticta (B: 1/2), Thryothorus nigricapillus Sclater, 1860 (HC: 0/6, B: 1/1) (Troglodytidae), Glyphorhynchus spirurus (HC: 1/10, B: 3/9), Xiphorhynchus susurrans (HC: 1/4, B: 0/2) (Dendrocolaptidae); Trogoniformes : Trogon rufus (B: 1/2) (Trogonidae); Piciformes : Melanerpes pucherani (B: 1/1) (Picidae).
Of the birds infested with chiggers, 32 of $39(82 \%)$ in the Hitoy Cerere BR, and 26 of 34 (77\%) in Barbilla NP were infested by $B$. sinnamaryi. Thus, the overall prevalence of chiggers in these localities reflects mainly the prevalence of this species.

Acknowledgements. We are grateful to the Ministerio del Ambiente y Energía de Costa Rica for permission to conduct our study (permission No. 106-2004-OFAU). We appreciate the effort of the staff of the field stations who kindly provided us with a logistical support. We owe a special debt to Bernardo Calvo Rodrígues for his friendship and permanent help. We thank Dr. Glen Coleman (Department of Parasitology, School of Veterinary Science, University of Queensland, Brisbane, Australia) for valuable comments and for the help in the preparation of the English text. This study was supported by the Grant of the Ministry of Science of the Russian Federation for State Support of Leading Scientific Schools Nos. SS1664.2003.4 and SS-5563.2006.4 (A.A. Stekol'nikov), and a grant No. LC06073 of the Ministry of Education, Youth and Sports of the Czech Republic (M. Capek).

\section{REFERENCES}

ARNOLD K.A. 1970: Notes on avian ectoparasites from Costa Rica. I. Acarina and Diptera. Rev. Biol. Trop. 16: 259-265.

BRENNAN J.M. 1969: Three new species of subgenus Parasecia Loomis (genus Fonsecia) from northeastern Brazil and a key to the included species (Acarina: Trombiculidae). J. Parasitol. 55: 662-666.

BRENNAN J.M., JONES E.K. 1960: Chiggers of Trinidad, B.W.I. (Acarina: Trombiculidae). Acarologia 2: 493-540.

BRENNAN J.M., REED J.T. 1974: The genus Eutrombicula in Venezuela (Acarina: Trombiculidae). J. Parasitol. 60: 699711.

BRENNAN J.M., VAN BRONSWIJK J.E.M.H. 1975: Parasitic mites of Surinam. XXI. New records of Surinam and certain French Guiana chiggers with the description of a new species of Loomisia Brennan \& Reed, 1972 (Acarina: Trombiculidae). J. Med. Entomol. 12: 243-249.

BRENNAN J.M., YUNKER C.E. 1966: The chiggers of Panama (Acarina: Trombiculidae) In: R.L. Wenzel and V.J. Tipton (Eds.), Ectoparasites of Panama. Field Museum Nat. Hist., Chicago, pp. 221-266.

Del Hoyo J., ElliotT A., Christie D. (Eds.) 2003: Handbook of the Birds of the World. Vol. 8: Broadbills to Tapaculos. Lynx Edicions, Barcelona, $845 \mathrm{pp}$.

Del Hoyo J., ElliotT A., Christie D. (Eds.) 2005: Handbook of the Birds of the World. Vol. 10: Cuckoo-shrikes to Thrushes. Lynx Edicions, Barcelona, $895 \mathrm{pp}$.

Del Hoyo J., ElliotT A., SARgATAL J. (Eds.) 2002: Handbook of the Birds of the World. Vol. 7: Jacarams to Woodpeckers. Lynx Edicions, Barcelona, 613 pp.

FlOCH H., FAURAN P. 1956: Sur deux espèces du genre "Trombicula" (Acariens, Trombiculidés) nouvelles pour la faune de la Guyane Française: "Trombicula alfreddugesi" (Oudemans, 1910) et "Trombicula sinnamaryi" n. sp. Arch. Inst. Pasteur Guyane Fr. 405, 7 pp.
Floch H., FAURAN P. 1957: Description de deux nouvelles espèces du genre "Trombicula" (Acariens, Trombiculidés) de Guyane Française. Arch. Inst. Pasteur Guyane Fr. 426, 6 pp.

FULLER H.S. 1952: The mite larvae of the family Trombiculidae in the Oudemans collection: taxonomy and medical importance. Zool. Verh. 18: 1-261.

GeEST J.C., LOOMIS R.B. 1968: Chiggers of the genus Pseudoschoengastia (Acarina: Trombiculidae) from Costa Rica. Contrib. Sci. (Los Angel.) 150, 49 pp.

GoFF M.L. 1988: A new species of chigger (Acari: Trombiculidae) from a vampire bat (Chiroptera: Desmodontidae) collected in Costa Rica. Int. J. Acarol. 14: 5-7.

GOFF M.L., LOOMIS R.B., WeLBOURN W.C., WRENN W.J. 1982: A glossary of chigger terminology (Acari: Trombiculidae). J. Med. Entomol. 19: 221-238.

SIXL W. 1969: Lebensweise und Verhalten von Ascoschöngastia latyshevi (Schluger) in Österreich (Acari, Trombiculidae). Oesterr. Akad. Wiss. Math.-Naturwiss. K1. Sitzungsber. Abt. I, 178: 271-293.

STILES F.G., SKUTCH A. 1989: A Guide to the Birds of Costa Rica. Christopher Helm, London, 511 pp.

SYCHRA O., LITERÁK I., ČAPEK M., HAVLÍČEK M. 2006: Chewing lice (Phthiraptera) from typical antbirds and ground antbirds (Passeriformes: Thamnophilidae, Formicariidae) from Costa Rica, with descriptions of three new species of the genera Formicaphagus and Myrsidea. Zootaxa 1206: 47-61.

WeBB J.P., LoOMIS R.B. 1971: Trombiculid mites of the genus Microtrombicula (Acarina) from Costa Rica. Contrib. Sci. (Los Angel.) 207, 15 pp.

WHARTON G.W., CARVER R.K. 1946: Food of nymphs and adults of Neoschöngastia indica (Hirst 1915). Science 104: 76-77. 